IOS Press

\title{
Anti-melanogenic compounds in Rubus croceacanthus
}

\author{
Michiyo Kubota $^{\mathrm{a}, 1}$, Takahiro Hosoya ${ }^{\mathrm{a}, 1}$, Syuichi Fukumoto ${ }^{\mathrm{b}}$, Tsuyoshi Miyagi ${ }^{\mathrm{c}}$ \\ and Shigenori Kumazawa ${ }^{\mathrm{a}, *}$ \\ ${ }^{a}$ Department of Food and Nutritional Sciences, University of Shizuoka, Yada, Suruga-ku, Shizuoka, Japan \\ ${ }^{\mathrm{b}}$ Pokka Sapporo Food and Beverage Ltd., Ebisu, Shibuya-ku, Tokyo, Japan \\ ${ }^{\mathrm{c}}$ Okinawa Prefectural Forest Resources Research Centre, Ohnaka, Nago, Okinawa, Japan
}

Submitted 6 November 2013; accepted 20 December 2013

\begin{abstract}
.
BACKGROUND: Rubus croceacanthus is a wild berry grown in Okinawa, Japan, and is consumed fresh because of its sweetness. In addition, this fruit is processed into jam and jelly, and used in food such as doughnuts in Okinawa. In our anti-melanogenesis screening program, the methanol extract of $R$. croceacanthus showed the whitening activity on B16 mouse melanoma cells.

OBJECTIVE: The aim of this study was to evaluate anti-melanogenetic activity of the extract of $R$. croceacanthus and find the active components in it.

METHOD: The anti-melanogenetic activity was examined using B16 cells. To reveal the mechanism underlying the antimelanogenic activity, the inhibition of tyrosinase which is the key enzyme in melanogenesis was measured. Furthermore, we analyzed the constituents of $R$. croceacanthus to investigate the active compounds.

RESULTS: The extract of $R$. croceacanthus had an anti-melanogenic effect in B16 cells. We found that this effect was caused by cyanidin-3-O-glucoside and pelargonidin-3-O-glucoside due to inhibit tyrosinase activity, resulting in decreasing melanin content in B16 cells. We also identified two ellagitannins, lambertianin $\mathrm{C}$ and sanguiin $\mathrm{H}-6$, which may contribute to the anti-melanogenic effect.
\end{abstract}

CONCLUSIONS: The fruit of $R$. croceacanthus has the anti-melanogenic activity and potential to be utilized as a skin-whitening agent.

Keywords: Rubus croceacanthus, Cyanidin-3-glucoside, Pelargonidin-3-glucoside, anti-melanogenesis, tyrosinase

\section{Introduction}

Rubus croceacanthus, which belongs to the Rosaceae family, is grown in Okinawa, Japan, and produces edible red aggregate berries. This fruit is consumed fresh or in products such as jam, jelly, and doughnuts in some areas of Okinawa. In our previous studies on $R$. croceacanthus, we isolated two anthocyanins, cyanidin-3-O-glucoside (Cy-3-glc) and pelargonidin-3-O-glucoside (Pg-3-glc), and also determined their quantities in the fruit (Fig. 1). $R$. croceacanthus has high anthocyanin contents; Pg-3-glc, in particular, was found at relatively higher levels than in other berries. In addition, we reported that this berry has potent antioxidant activity compared to raspberry (Rubus idaeus), one of the most popular fruits in the genes Rubus [1].

\footnotetext{
${ }^{1}$ These authors contributed equally to this work.

${ }^{*}$ Corresponding author: Shigenori Kumazawa, Department of Food and Nutritional Sciences, University of Shizuoka, 52-1 Yada, Suruga-ku, Shizuoka 422-8526, Japan. Tel./Fax: +81 54264 5523; E-mail: kumazawa@u-shizuoka-ken.ac.jp.
} 


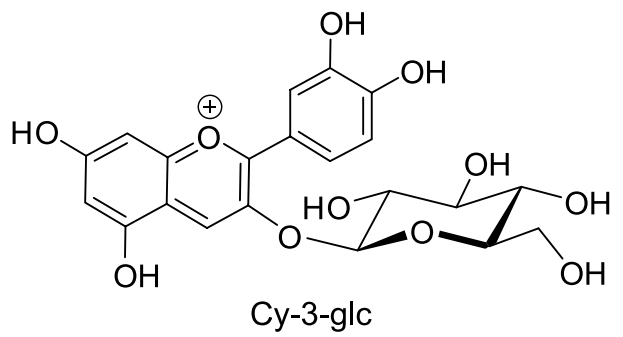<smiles>OCC(O)C1OC2OC(O)C(O)C1Oc1cc3c(O)cc(O)cc3[o+]c12</smiles>

Pg-3-glc

Fig. 1. The structures of the anthocyanins in $R$. croceacanthus. Cy-3-glc, cyanidin-3-O-glucoside; Pg-3-glc, pelarugonidin-3-O-glucoside.

Anthocyanidins are pigments responsible for the blue, purple, orange, and red colours in plants, and exist primarily in their glycoside forms, referred to as anthocyanins, which are present in the tissues of coloured fruits, vegetables, and flowers [2, 3]. Anthocyanidins and anthocyanins are believed to have various biological effects that are beneficial to human health, including antioxidant, anticarcinogenic, antiobesity, and antidiabetic activities; induction of apoptosis; and prevention of DNA damage $[4,5]$. Because of these effects, the regular consumption of anthocyanin-rich foods is thought to be associated with a reduced risk of developing lifestyle-related diseases [6, 7].

Berries, which contain large amount of anthocyanins, are small fleshy fruits, and are typically consumed fresh or in products such as juice, jam, jelly, wine, and syrup. The content of anthocyanins in berries depends on the species, variety, cultivation, region, weather conditions, ripeness, and storage time $[8,9]$. Along with anthocyanins, berries contain other beneficial components, such as vitamin $\mathrm{C}$ (ascorbic acid), phenolic acids, tannins, stilbenes, and flavonoids.

In our screening of anti-melanogenic activity, we found that the methanol extract of $R$. croceacanthus showed the whitening effect with the assay of B16 mouse melanoma cells. In this study, we demonstrated the anti-melanogenic activity of the methanol extract of $R$. croceacanthus. In order to identify the active components, the ability of two major anthocyanins of $R$. croceacanthus to inhibit melanogenesis in the B16 mouse melanoma cells, and mushroom tyrosinase, which is the key enzyme in melanogenesis was evaluated. Furthermore, we investigate ascorbic acid and/or some tannins of $R$. croceacanthus as potential candidates for skin whitening. Ascorbic acid was quantified using high performance liquid chromatography (HPLC), and two ellagitannins were isolated and identified by spectroscopic analyses.

\section{Materials and methods}

\subsection{Chemicals and reagents}

B16 melanoma 4A5 cells were purchased from Riken cell Bank (Tsukuba, Japan). Dulbecco's modified Eagle's medium (phenol red-free) (DMEM), L-glutamine and L-tyrosine were purchased from Wako Pure Chemical Industries Ltd. (Osaka, Japan). Fetal bovine serum (FBS) and penicillin-streptomycin were purchased from Gibco Invitrogen Co., Ltd. (Carlsbad, CA). Arbutin and mushroom tyrosinase was purchased from Sigma Chemical Co. (St. Louis, MO). The 5-[2,4-Bis(sodiooxysulfonyl)phenyl]-3-(2-methoxy-4-nitrophenyl)-2-(4-nitrophenyl)-2Htetrazole-3-ium (WST-8) was purchased from Dojindo Laboratories (Kumamoto, Japan). Cyanidin-3-glucoside chloride and pelargonidin-3-glucoside chloride were purchased from Funakoshi Co., Ltd. (Tokyo, Japan). Kojic acid was purchased from Tokyo Chemical Industry Co., Ltd. (Tokyo, Japan).

\subsection{Plant material}

Fruits of $R$. croceacanthus (300 g) were donated by Kinjo farm (Okinawa, Japan) in March 2011. Fruits of $R$. idaeus $(50 \mathrm{~g})$ were obtained from SICOLY (St. Laurent d'Agny, France). The fresh berry samples were homogenized in liquid nitrogen and lyophilized using an EYELA FD-5N freeze-dryer (Tokyo Rikakikai, Co. Ltd., Tokyo, Japan). All freeze-dried powder was stored in a freezer $\left(-20^{\circ} \mathrm{C}\right)$ until analysis. 


\subsection{Measurement of melanogenesis in cultured B16 melanoma cells}

B16 cells were maintained in DMEM containing 10\% FBS, L-glutamine (4 mM), penicillin (100 U/mL) and streptomycin $(100 \mu \mathrm{g} / \mathrm{mL})$ without phenol red at $37^{\circ} \mathrm{C}$ in a humidified atmosphere of $5 \% \mathrm{CO}_{2}$ and $95 \%$ air. The cells $\left(1.5 \times 10^{4}\right.$ cell $\left./ \mathrm{mL}\right)$ were seeded in each well of a 48 -well plate, and preincubated for $24 \mathrm{~h}$. After preincubation, the cells were cultured in fresh DMEM added test sample dissolved in dimethyl sulfoxide (DMSO) for $48 \mathrm{~h}$. The medium was then replaced with fresh DMEM containing the test samples, and the cells were incubated.

After $72 \mathrm{~h}$ of incubation, the medium was replaced with DMEM containing 5\% WST-8, and the cells were cultured for $1 \mathrm{~h}$ to measure the cell viability. The absorbance at $450 \mathrm{~nm}$ was determined using a microplate reader, and compared with that of the control.

The cells were then washed with phosphate buffered saline (PBS), dissolved in $100 \mu \mathrm{L}$ of $1 \mathrm{M} \mathrm{NaOH}$, and heated at $90^{\circ} \mathrm{C}$ for $1 \mathrm{~h}$. The melanin content was calculated by comparing the absorbance at $400 \mathrm{~nm}$ of the sample with that of the control. Arbutin was applied used as a positive control.

\subsection{Measurement of tyrosinase inhibition}

The inhibition of tyrosinase activity was assayed using a spectroscopic method. In this assay, $50 \mu \mathrm{L}$ of test samples and $50 \mu \mathrm{L}$ of $100 \mathrm{U} / \mathrm{mL}$ tyrosinase were added to a 96 -well plate. Then, $100 \mu \mathrm{L}$ of $2.0 \mathrm{mM}$ L-tyrosine was added to each well, and the contents of each well were mixed. After incubation at $37^{\circ} \mathrm{C}$ for $30 \mathrm{~min}$, the amount of dopachrome product was determined using the absorbance at $475 \mathrm{~nm}$. The tyrosinase inhibition of each test sample was calculated as follows: \% inhibition $=\left\{\left(\mathrm{A}-\mathrm{A}^{\prime}\right)-\left[\left(\mathrm{B}-\mathrm{B}^{\prime}\right)-\left(\mathrm{C}-\mathrm{C}^{\prime}\right)\right] / \mathrm{A}-\mathrm{A}^{\prime}\right\} \times 100$; where $\mathrm{A}$ is the $\mathrm{OD}_{475}$ of enzyme and substrate; $\mathrm{A}^{\prime}$ is the $\mathrm{OD}_{475}$ of substrate; $\mathrm{B}$ is the $\mathrm{OD}_{475}$ of enzyme, substrate and test sample; $\mathrm{B}^{\prime}$ is the $\mathrm{OD}_{475}$ of enzyme and test sample; $\mathrm{C}$ is the $\mathrm{OD}_{475}$ of substrate and test sample; and $\mathrm{C}^{\prime}$ is the $\mathrm{OD}_{475}$ of test sample. Kojic acid was used as a positive control.

\subsection{Quantification of ascorbic acid}

The ascorbic acid content of each type of berry was determined by HPLC analysis. The freeze-dried sample of each berry $(10 \mathrm{mg})$ was added to $200 \mu \mathrm{L}$ of $5 \%$ metaphosphoric acid and $800 \mu \mathrm{L}$ of distilled water. The solution was mixed and the supernatant was recovered by centrifugation at $500 \times g$ for $10 \mathrm{~min}$. Then, $100 \mu \mathrm{L}$ of $1 \%$ dithiothreitol and $1 \mathrm{~mL}$ of $\mathrm{KH}_{2} \mathrm{PO}_{4}$ buffer $(\mathrm{pH} 7.4)$ were added to the supernatant and the solution was reacted at $30^{\circ} \mathrm{C}$ for $20 \mathrm{~min}$ to obtain the L-ascorbic acid by reducing the L-dehydroascorbic acid. After the reaction, the solution was filtered through a $0.45 \mu \mathrm{m}$ membrane filter (Kanto Chemicals, Tokyo, Japan) prior to the injection of $20 \mu \mathrm{L}$ into an analytical HPLC system. HPLC analysis was carried out on a machine equipped with a Jasco PU-980 pump, LG-2080-02 gradient unit, UV-2075 plus detector, DG-2080-53 degasser, and Capcell Pak UG120 reversed phase column (4.6 mm i.d. $\times 250 \mathrm{~mm}, 5 \mu \mathrm{m}$; Shiseido) with $20 \mathrm{mM}$ phosphoric acid. The flow rate was $1 \mathrm{~mL} / \mathrm{min}$ and the detection wavelength was $254 \mathrm{~nm}$.

\subsection{Extraction and isolation of ellagitannnins}

The freeze-dried powder of $R$. croceacanthus $(53.4 \mathrm{~g}$ ) was stirred and sonicated in $300 \mathrm{~mL}$ of $80 \%$ methanol containing $0.5 \%$ acetic acid. The solution was filtered using a Büchner funnel. The filter residue was re-extracted six times until a clear solution was obtained. After extracting, the filtrates $1.8 \mathrm{~L}$ were evaporated under reduced pressure to get the dried crude extract. The extract was dissolved in about $100 \mathrm{~mL}$ of water, and passed through a DIAION HP-20 $(60 \times 300 \mathrm{~mm}$; Mitsubishi Chemicals, Tokyo, Japan $)$ column prewashed with $0.1 \%$ trifluoro acetic acid (TFA) in water. The extract was then eluted with a gradient of $0-50 \%$ and $100 \%$ methanol containing $0.1 \%$ TFA [frs. 1-3, $\mathrm{H}_{2} \mathrm{O}$ eluate $(2.0 \mathrm{~L})$; frs. 4-8, $10 \%$ methanol eluate (1.5 L); frs. 9-10, $20 \%$ methanol eluate $(1.0 \mathrm{~L})$; frs. 11-14, 30\% methanol eluate $(2.0 \mathrm{~L})$; frs. $15-18,40 \%$ methanol eluate $(3.0 \mathrm{~L})$; frs. $19-21,50 \%$ methanol eluate $(1.0 \mathrm{~L})$; and frs. $22-23,100 \%$ methanol eluate $(0.8 \mathrm{~L})]$. These fractions were concentrated using a rotary evaporator to obtain the dried fractions. 
Preparative HPLC was carried out on a machine equipped with a Jasco PU-2086 plus pump and UV-970 detector (Tokyo, Japan) using Capcell Pak C18 UG120 reversed phase column $(20 \mathrm{~mm}$ i.d. $\times 250 \mathrm{~mm}$, i.d., $5 \mu \mathrm{m}$; Shiseido, Tokyo, Japan). Fr. 16 in methanol containing 0.5\% TFA was analysed using an isocratic elution method with $0.1 \%$ TFA in $13 \%$ acetonitrile at a flow rate of $12 \mathrm{~mL} / \mathrm{min}$ to get the compound $1(6.74 \mathrm{mg})$ and $2(7.73 \mathrm{mg})$. The compounds were detected at $270 \mathrm{~nm}$ wavelength.

\subsection{Identification of ellagitannins}

The compounds isolated by preparative HPLC were identified using nuclear magnetic resonance (NMR), mass spectrometry (MS), and circular dichroism (CD). NMR measurements were performed in acetone- $d_{6}$ using an AVANCE III (400 MHz) spectrometer (Bruker BioSpin, Rheinstetten, Germany). The $1 \mathrm{D}{ }^{1} \mathrm{H},{ }^{13} \mathrm{C}$, and TOCSY and the $2 \mathrm{D}$ COSY and HSQC spectra were obtained for identification. ESI-MS was executed on an LCQ ion trap mass spectrometer (Thermo Fisher Scientific K.K., MA, USA). The operating parameters were: source voltage, $5 \mathrm{kV}$; ES capillary voltage, $10 \mathrm{~V}$; capillary temperature, $200^{\circ} \mathrm{C}$. MALDI-TOF MS was performed on a Bruker Daltonics ultraflex (Bruker Daltonics, Billerica, USA) and 2,5-dihydroxybenzoic acid in 90\% methanol was used as a matrix. CD spectra were recorded using a Jasco J-600 spectorometer (Tokyo, Japan).

\section{Results}

\subsection{Anti-melanogenic activity of $R$. croceacanthus in cultured B16 melanoma cells}

The anti-melanogenic effects of the $80 \%$ methanol extract of $R$. croceacanthus were determined in B16 mouse melanoma cells. Figure 2 shows the melanin content in B16 cells. In order to evaluate the effect of the extract, the melanin content of the treated cells and the corresponding cell viability was compared with that of the control. $R$. idaeus, which contains two main anthocyanins, cyanidin-3-sophoroside and cyanidin-3-glucoside, was used as a comparison.

The melanin production was decreased due to treatment with the extracts of $R$. croceacanthus and $R$. idaeus in a dose-dependent manner. The decrease in melanin content after treatment with the two extracts, at the concentration $200 \mu \mathrm{g} / \mathrm{mL}$, were $67.0 \pm 2.5 \%$ and $63.2 \pm 2.5 \%$, respectively, without any effect on the cell viability. These results indicate the whitening effect of the berry extracts on B16 cells.
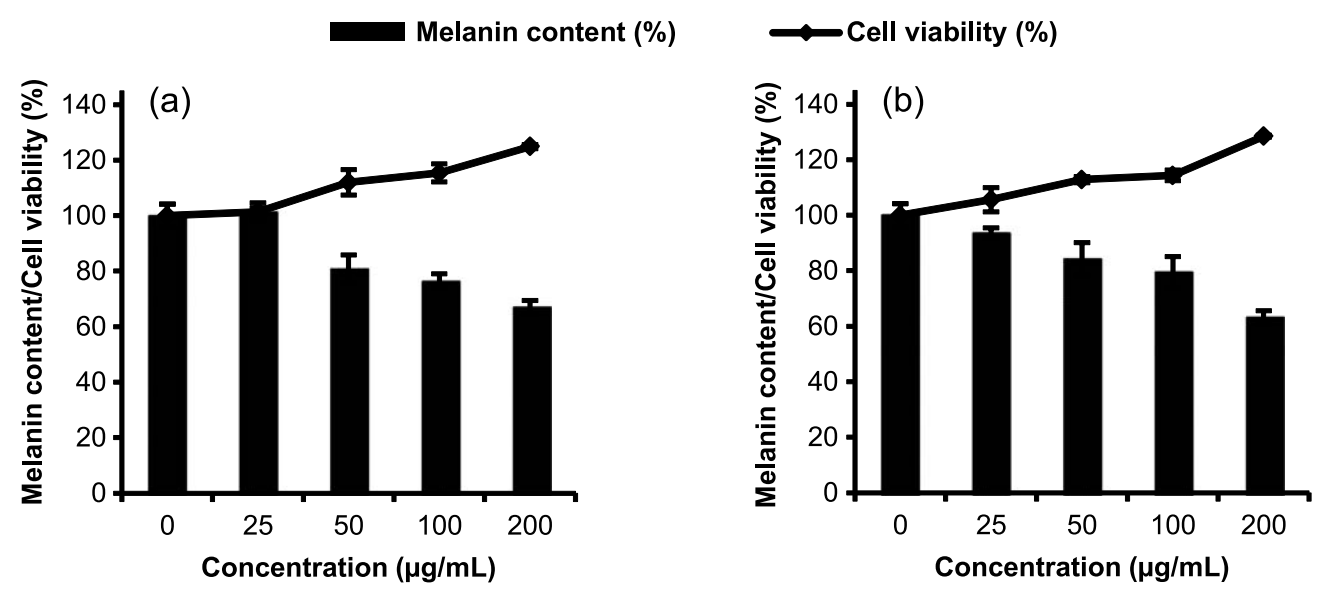

Fig. 2. The anti-melanogenic activity of the methanol extracts of $R$. croceacanthus (a) and $R$. idaeus (b) in B16 mouse melanoma cells. The solid column shows the melanin content in B16 cells and no cytotoxicity at all concentrations. The melanin content was normalized to the number of cells. Samples were assayed in triplicate. Bars represent standard deviation. 


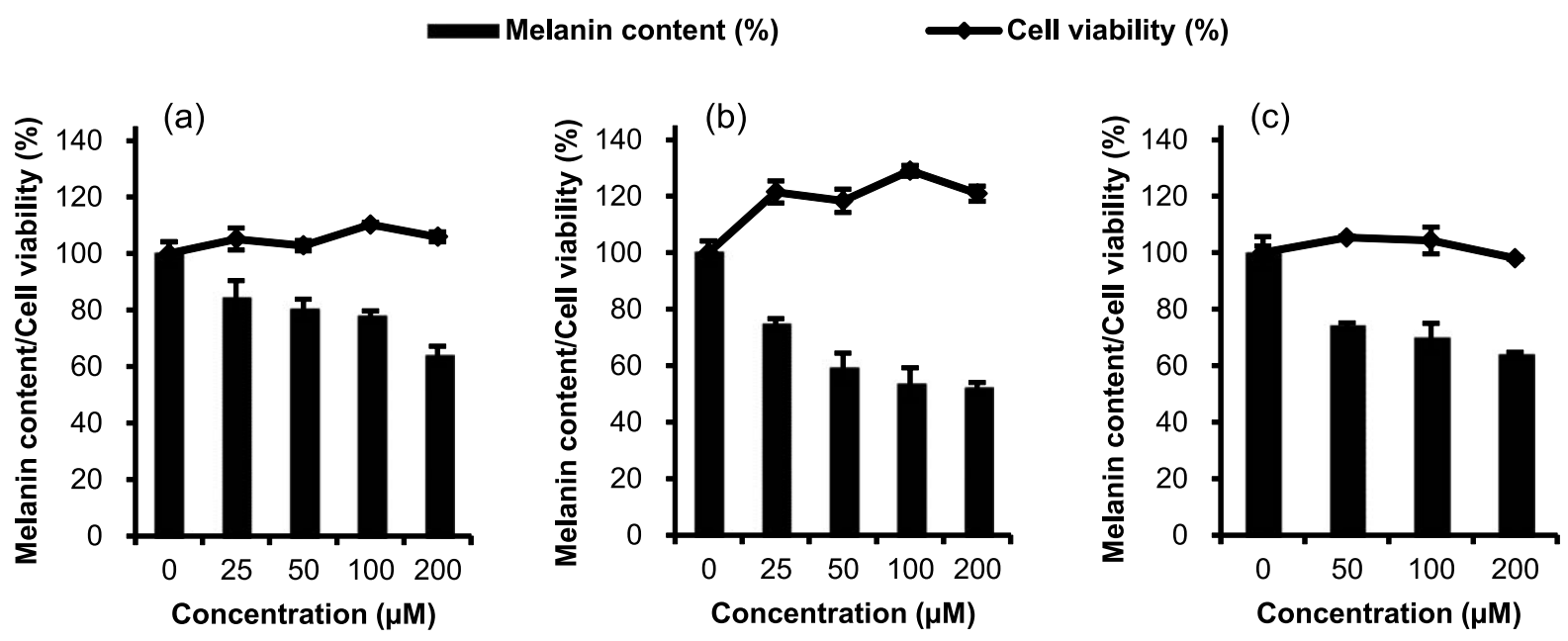

Fig. 3. The anti-melanogenic activity of anthocyanins (Cy-3-glc (a) and Pg-3-glc (b)) and arbutin as a positive control (c) in B16 mouse melanoma cells. The solid column shows the melanin content in B16 cells and no cytotoxicity at all concentrations. The melanin content was normalized to the number of cells. Compounds were assayed in triplicate. Bars represent standard deviation.

\subsection{Anti-melanogenic activity of anthocyanins}

Cy-3-glc and Pg-3-glc, which are the major anthocyanins in R. croceacanthus, were tested for anti-melanogenic activity using B16 melanoma cells. Both the anthocyanins showed dose-dependent anti-melanogenic activity in the B16 cells. As shown in Fig. 3, the whitening activity of Pg-3-glc was greater than that of Cy-3-glc, whose effects were greater than or equivalent to those of arbutin, which is used as a major cosmetic additives. Our results indicated that the major anti-melanogenic compounds in $R$. croceacanthus were Cy-3-glc and/or Pg-3-glc.

\subsection{Measurement of tyrosinase inhibition}

To investigate the mechanism underlying the anti-melanogenic activity, the inhibitory effect of the extracts on tyrosinase, which is a key enzyme in melanogenesis, was assayed. Tyrosinase catalyzes the oxidization of L-tyrosine to 3,4-dihydroxyphenylalanine (DOPA) and the oxidization of DOPA to dopaquinone, the first two rate-limiting steps in melanin synthesis pathway [10]. Therefore, melanogenesis can be inhibited by the activity of tyrosinase and/or expression of tyrosinase in cells.

Figure 4 illustrates the result of the tyrosinase inhibitory assay in terms of the percentage of tyrosinase inhibition of each berry extract and each anthocyanin. Although the inhibitory effects of each berry extract on melanin production

(a)

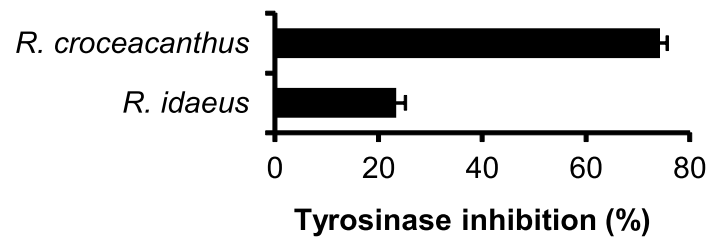

(b)

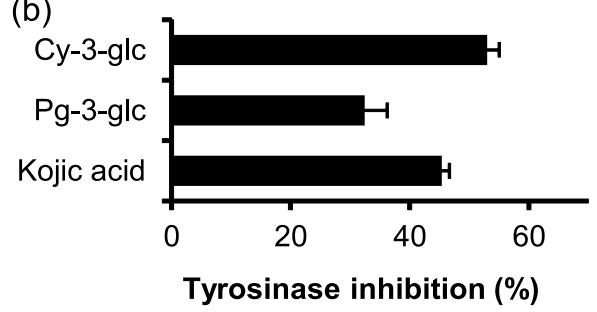

Fig. 4. The tyrosinase inhibitory activity of the methanol extracts of $R$. croceacanthus and $R$. idaeus at a concentration of $250 \mu \mathrm{g} / \mathrm{mL}$ (a), and Cy-3-glc, Pg-3-glc, and kojic acid as a positive control at a concentration of $25 \mu \mathrm{M}$ (b). Samples were assayed in triplicate. Bars represent standard deviation. 
Table 1

Tyrosinase inhibitory activity

\begin{tabular}{lc}
\hline & $\mathrm{IC}_{50}(\mu \mathrm{g} / \mathrm{mL})$ \\
\hline$R$. croceacanthus & 101.1 \\
$R$. idaeus & $>1000$ \\
Cy-3-glc & 10.2 \\
Pg-3-glc & 21.5 \\
Kojic acid & 4.0
\end{tabular}

Table 2

Ascorbic acid content in each berry

\begin{tabular}{lc}
\hline & Ascorbic acid (mg/100g fresh weight) \\
\hline R. croceacanthus & $6.56 \pm 0.21$ \\
$R$. idaeus & $22.83 \pm 0.30$ \\
\hline
\end{tabular}

Each value is expressed as mean \pm standard deviation $(n=3)$.

in B16 mouse melanoma cells were similar, $R$. croceacanthus had greater anti-tyrosinase activity than $R$. idaeus. The two anthocyanins also inhibited the tyrosinase activity; particularly, Cy-3-glc showed a higher inhibition than kojic acid, which is a well-known tyrosinase inhibitor. The $\mathrm{IC}_{50}$ values for Cy-3-glc, Pg-3-glc, and kojic acid were 22.6, 49.7, and $28.3 \mu \mathrm{M}$, respectively (Table 1 ).

\subsection{Quantification analysis of ascorbic acid}

The ascorbic acid content of each berry was determined by including the levels of both L-ascorbic acid, which is reduced form, and L-dehydroascorbic acid, which is the oxidized form. In other words, L-dehydroascorbic acid was reduced by dithiothreitol, which is a reducing agent, and L-ascorbic acid was detected by analytical HPLC at $254 \mathrm{~nm}$. The quantitative analysis of the ascorbic acid was carried out using the calibration curves, which were constructed and tested for linearity. A good linearity $\left(r^{2}>0.999\right)$ was observed for the relationship of the peak area and concentrations over the tested range $(0.063-4.0 \mu \mathrm{g} / \mathrm{mL})$. The limit of detection (LOD) value and the limit of quantitation (LOQ) value were $0.016 \mu \mathrm{g} / \mathrm{mL}$ and $0.052 \mu \mathrm{g} / \mathrm{mL}$, respectively. Table 2 shows the content of ascorbic acid in $R$. croceacanthus and $R$. idaeus as $\mathrm{mg}$ per $100 \mathrm{~g}$ fresh fruit weight. Although $R$. idaeus had approximately 3.5 times the concentration of ascorbic acid found in $R$. croceacanthus, the amount of ascorbic acid was very low compared with that in other berries.

\subsection{Identification of ellagitaninns}

The fruit extract of $R$. croceacanthus were prepared by extraction with $80 \%$ methanol containing $0.5 \%$ acetic acid at room temperature. The extracts were separated using column chromatography and two compounds, which were observed as the major phenolic compounds other than the anthocyanins in $R$. croceacanthus in the HPLC spectrum at $270 \mathrm{~nm}$, were isolated by preparative HPLC. The two compounds, 1 and 2, isolated from $R$. croceacanthus, were identified using NMR, MS, and CD (Fig. 5). These compounds contained fragment ion at $\mathrm{m} / \mathrm{z}$ 301, which was detected using ESI-MS by the positive ionization, indicating the presence of a hexahydroxydiphenoyl (HHDP) group, which is one of the groups present in the ellagitannins. Compound 1 had a $[M+\mathrm{Na}]^{+}$ion peak at $m / z 2827$, which was detected by the MALDI-TOF-MS, indicating that 1 is an ellagitannin trimer. Compound 2 had a $[M+\mathrm{Na}]^{+}$ion peak at $m / z$. 1893, which was detected by the ESI-MS, indicating that 2 is an ellagitannin dimer. The NMR and MS spectra were compared with those spectra of ellagitannin reported previously [11]. These spectra of 1 were similar to those of rubusuaviin $\mathrm{C}$ or lambertianin $\mathrm{C}$. The ${ }^{1} \mathrm{H}$ NMR spectrum of 1 showed three anomeric protons at $\delta 6.52(\mathrm{~J}=4.0 \mathrm{~Hz}$; $\mathrm{H}-1), \delta 5.99\left(J=8.6 \mathrm{~Hz} ; \mathrm{H}-1^{\prime}\right)$, and $\delta 6.17\left(J=9.0 \mathrm{~Hz} ; \mathrm{H}-1^{\prime \prime}\right)$, suggesting that glucose $\mathrm{I}$ is in the $\alpha$-configuration 


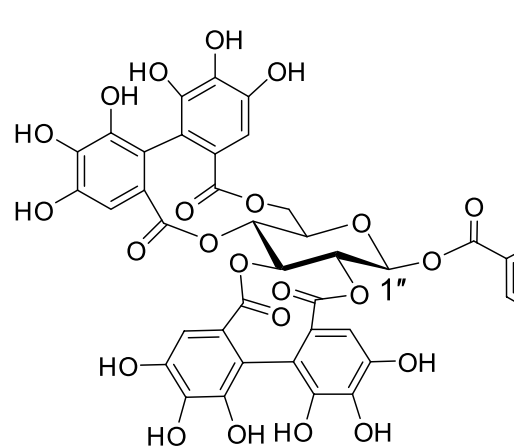

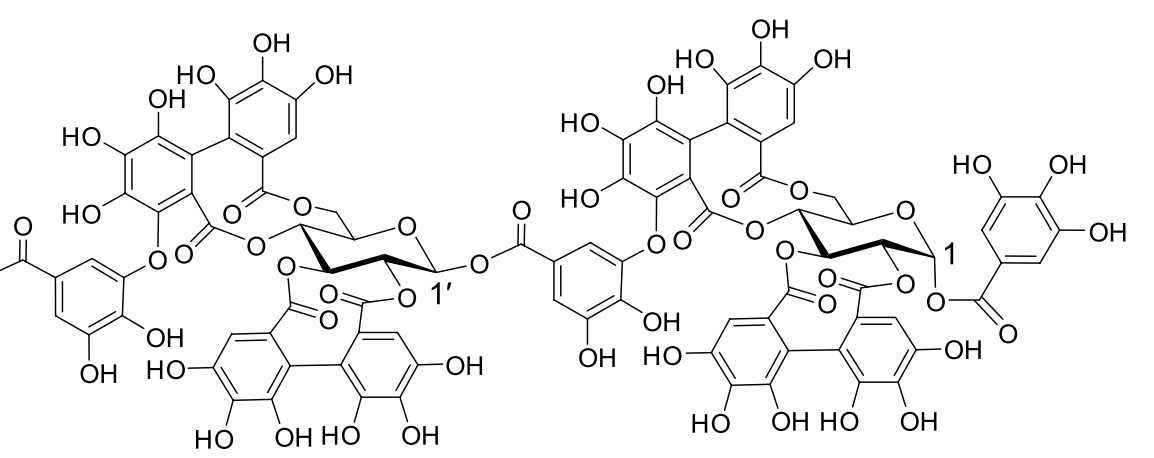

1 : Lambertianin C

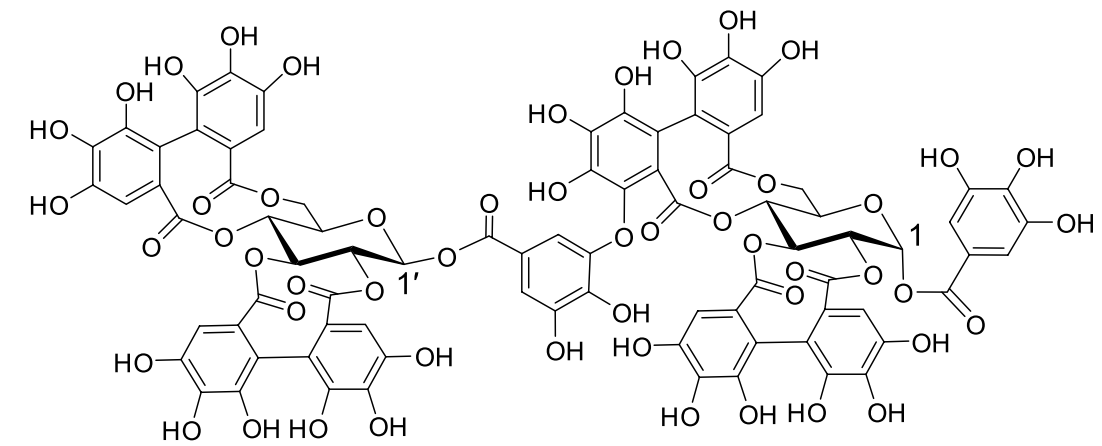

2 : Sanguiin $\mathrm{H}-6$

Fig. 5. The structures of ellagitannins isolated from $R$. croceacanthus.

and glucose II and III are in the $\beta$-configuration, which is consistent with the previously reported structure of lambertianin C [11]. The NMR and MS spectra of 2 were similar to those of sanguiin $\mathrm{H}-6$ or lambertianin A. The ${ }^{1} \mathrm{H}$ NMR spectrum of 2 showed two anomeric protons at $\delta 6.52(J=4.0 \mathrm{~Hz} ; \mathrm{H}-1)$ and $\delta 6.15\left(\mathrm{~J}=8.8 \mathrm{~Hz} ; \mathrm{H}^{\prime} 1^{\prime}\right)$, suggesting that glucose I is in the $\alpha$-configuration and glucose II is in the $\beta$-configuration, which is consistent with the previously reported structure of sanguiin H-6 [11]. The CD spectra of compounds 1 and 2 were also compared and identified as those of lambertianin $\mathrm{C}$ and sanguiin H-6, respectively. These components have been reported to be present in raspberry (Rubus idaeus) and blackberry (Rubus fruticosus), which are popular berry fruits belonging to the Rubus genus [11].

\section{Discussion}

The amount of melanin produced by B16 melanoma cells decreased in a dose-dependent manner on treatment with the methanol extract of $R$. croceacanthus. The major components of $R$. croceacanthus are two anthocyanins, Cy-3-glc and Pg-3-glc [1]. Therefore, the anti-melanogenic activity of these two anthocyanins was also evaluated. The two anthocyanins had an inhibitory effect on melanin production in the B16 cells, and their activities were stronger than or equivalent to those of arbutin, which was used as a positive control. This result suggests that the anthocyanins significantly contribute to the anti-melanogenic properties of $R$. croceacanthus. To investigate the mechanism underlying the inhibition of the melanogenesis, we examined their effects on mushroom tyrosinase. The extract of $R$. croceacanthus and two major anthocyanins from $R$. croceacanthus demonstrated anti-tyrosinase activity. 
This indicated that the anthocyanins in $R$. croceacanthus probably contribute to tyrosinase inhibition, and that this effect facilitates the inhibition of melanin production in B16 mouse melanoma cells. Tyrosinase is a metalo-enzyme containing copper, which acts on the oxidative reaction at the active site [12]. It is considered that the copper binds with histidine residues at the active site. Since anthocyanin is reported to chelate some metal ions [13], anthocyanin may act on the active site. Moreover, antioxidants are considered to inhibit the various oxidative steps of melanin formation because tyrosinase is an oxidase, and free radicals have been reported to upregulate the levels of tyrosinase mRNA [14]. Accordingly, the antioxidant activity may also contribute to the reduction of pigmentation by the anthocyanins.

The extracts of $R$. idaeus as well as $R$. croceacanthus had anti-melanogenic activity. However, the inhibition of the tyrosinase enzyme activity by $R$. idaeus was about three times weaker than that by $R$. croceacanthus at a concentration of $250 \mu \mathrm{g} / \mathrm{mL}$. Our findings indicated that Cy-3-glc is present in $R$. idaeus, but its levels were about eleven times less than that in $R$. croceacanthus, as reported in a previous study [1]. Therefore, the anti-melanogeneic activity of $R$. idaeus is probably mediated by the inhibition of tyrosinase expression. Furthermore, it is possible that the anthocyanins in $R$. idaeus do not fully influence the inhibition of melanin production, and perhaps other compounds contribute the inhibitory effect on melanogenesis.

Ascorbic acid is widely distributed in various plants including berry fruit. It is a major antioxidant and a skin whitening agent [15]. Therefore, we quantified the ascorbic acid in the two types of berry fruits to identify the active ingredients responsible for their anti-melanogenic effect. The ascorbic acid content of $R$. idaeus was higher than that of $R$. croceacanthus. However, ascorbic acid is probably not the main active compound of these berries because its content of in $R$. idaeus was about 2.5-4 times lower than that in strawberry (Fragaria $\times$ ananassa), which belong to the Rosaceae family but not to the Rubus genus [16].

In addition to the quantification of ascorbic acid, we isolated the main constituents from $R$. croceacanthus to find the effective compounds other than the anthocyanins. Compounds 1 and 2 were detected and purified by preparative HPLC. Structural analysis of these compounds identified them as the ellagitannins, lambertianin C and sanguiin H-6. The ellagitannins have been found in abundance in the fruit of $R$. idaeus [11]. Ellagitannins belong to a group of hydrolysable tannins and are polymers of hexahydroxydiphenolic acid, which is a dimeric form of gallic acid. It is believed that hydrolysable tannins including ellagitannins have antioxidant and anti-melanogenic activities, and the melanogenesis-inhibitory activity is due to the inhibition of the expression of MITF, followed by a decrease in the expression of tyrosinase and TRP-1 [17]. Therefore, the ellagitannins in berries may contribute to the reduction of pigmentation. Additionally, ellagitannins are readily hydrolysed under acidic or basic conditions to release ellagic acid because hexahydroxydiphenolic acids can lactonize to form ellagic acid, which is found in a variety of plants. Ellagic acid has been reported to suppress melanogenesis by reacting with activated melanocytes [18]. The results of this study suggest that the ellagic acids were formed by hydrolysis of the ellagitannins in the B16 melanoma cells, which in turn facilitated the anti-melanogenic effect of $R$. idaeus. This hypothesis is consistent with our observations indicating that the inhibitory effect of the extract of $R$. idaeus on the melanogenesis was higher than that expected for its corresponding anti-tyrosinase activity.

Proanthocyanidins belong to a group of condensed tannins and are classified as procyanidins and prodelphinidins, according to the flavan-3-ol units. Proanthocyanidins are present in Rubus fruits and are found in R. idaeus, but the levels of the proanthocyanidins are much lower than those of the ellagitannins [19]. Since procyanidins are effective inhibitors of tyrosinase [20], they may also contribute to the anti-melanogenic activity of $R$. idaeus.

In conclusion, the fruit of $R$. croceacanthus and the two main anthocyanins present in them inhibited the melanin production in B16 melanoma cells. The present results are the first report on the anti-melanogenic activity of Cy-3-glc and Pg-3-glc. The fruit of $R$. croceacanthus and the two main anthocyanins in them also inhibited tyrosinase activity. These results indicate that anthocyanins significantly contribute to the anti-melanogenic effect of $R$. croceacanthus, which is facilitated by the inhibition of enzymatic activity of tyrosinase. The fruit of $R$. croceacanthus therefore has the potential to be utilized as a skin-whitening agent.

\section{Acknowledgments}

We thank Atsuo Kinjo (Kinjo farm, Okinawa, Japan) for providing the R. croceacanthus. 


\section{References}

[1] Kubota M, Ishikawa C, Sugiyama Y, Fukumoto S, Miyagi T, Kumazawa S. Anthocyanins from the fruits of Rubus croceacanthus and Rubus sieboldii, wild berry plants from Okinawa, Japan. Journal of Food Composition and Analysis. 2012;28:179-82.

[2] Koponen JM, Happonen AM, Mattila PH, Törrönen AR. Contents of anthocyanins and ellagitannins in selected foods consumed in Finland. Journal of Agricultural and Food Chemistry. 2007;55:1612-9.

[3] Wu X, Beecher GR, Holden JM, Haytowitz DB, Gebhardt SE, Prior RL. Concentrations of anthocyanins in common foods in the United States and estimation of normal consumption. Journal of Agricultural and Food Chemistry. 2006;54:4069-75.

[4] Tsuda T. Dietary anthocyanin-rich plants: Biochemical basis and recent progress in health benefits studies. Molecular Nutrition and Food Research. 2012;56:159-70.

[5] Wang LS, Stoner GD. Anthocyanins and their role in cancer prevention. Cancer Letters. 2008;269:281-90.

[6] Harborne JB, Williams CA. Advances in flavonoid research since 1992. Phytochemistry. 2000;55:481-504.

[7] Zafra-Stone S, Yasmin T, Bagchi M, Chatterjee A, Vinson JA, Bagchi D. Berry anthocyanins as novel antioxidants in human health and disease prevention. Molecular Nutrition and Food Research. 2007;51:675-83.

[8] Aherne SA, O’Brien NM. Dietary flavonols: Chemistry, food content, and metabolism. Nutrition. 2002;18:75-81.

[9] Haffner K, Rosenfeld HJ, Skrede G, Wang L. Quality of red raspberry Rubus idaeus L. cultivars after storage in controlled and normal atmospheres. Postharvest Biology and Technology. 2002;24:279-89.

[10] Hearing V, Jiménz M. Mammalian tyrosinase-the critical regulatory control point in melanocyte pigmentation. International Journal of Biochemistry. 1987;19:1141-7.

[11] Gasperotti M, Masuero D, Vrhovsek U, Guella G, Mattivi F. Profiling and accurate quantification of Rubus ellagitannins and ellagic acid conjugates using direct UPLC-Q-TOF HDMS and HPLC-DAD analysis. Journal of Agricultural and Food Chemistry. 2010;58:4602-16.

[12] Jackman MP, Huber H, Hajnal A, Lerch K. Stabilization of the oxy form of tyrosinase by a single conservative amino acid substitution. Biochemical Journal. 1992;282:915-8.

[13] Castañeda-Ovando A, Pacheco-Hernández MDL, Páez-Hernández ME, Rodríguez JA, Galán-Vidal CA. Chemical studies of anthocyanins: A review. Food Chemistry. 2009;113:859-71.

[14] Rout S, Banerjee R. Free radical scavenging, anti-glycation and tyrosinase inhibition properties of a polysaccharide fraction isolated from the rind from Punica granatum. Bioresource Technology. 2007;98:3159-63.

[15] Choi YK, Rho YK, Yoo KH, Lim YY, Li K, Kim BJ, Seo SJ, Kim MN, Hong CK, Kim DS. Effects of vitamin C vs. multivitamin on melanogenesis: Comparative study in vitro and in vivo. International Journal of Dermatology. 2010;49:218-26.

[16] Aoyama F, Nomura H. Research on the quantity of the vitamin C content in strawberries by off-season culture and by normal culture. Journal of Japanese Society of Food and Nutrition. 1972;25:105-7.

[17] Manosroi A, Jantrawut P, Ogihara E, Yamamoto A, Fukatsu M, Yasukawa K, Tokuda H, Suzuki N, Manosroi J, Akihisa T. Biological activities of phenolic compounds and triterpenoids from the galls of Terminalia chebula. Chemistry and Biodiversity. 2013;10:1448-63.

[18] Shimogaki H, Tanaka Y, Tamai H, Masuda M. In vitro and in vivo evaluation of ellagic acid on melanogenesis inhibition. International Journal of Cosmetic Science. 2000;22:291-303.

[19] Beekwilder J, Jonker H, Meesters P, Hall RD, van der Meer IM, Ric de Vos CH. Antioxidants in raspberry: On-line analysis links antioxidant activity to a diversity of individual metabolites. Journal of Agricultural and Food Chemistry. 2005;53:3313-20.

[20] Shoji T, Masumoto S, Moriichi N, Kobori M, Kanda T, Shinmoto H, Tsushida T. Procyanidin trimers to pentamers fractionated from apple inhibit melanogenesis in B16 mouse melanoma cells. Journal of Agricultural and Food Chemistry. 2005;53:6105-11. 